\title{
Semiclassical theory of chaotic quantum resonances
}

\author{
T. Micklitz ${ }^{1}$ and A. Altland ${ }^{2}$ \\ ${ }^{1}$ Dahlem Center for Complex Quantum Systems and Institut für Theoretische Physik, Freie Universität Berlin, 14195 Berlin, Germany \\ ${ }^{2}$ Institut für Theoretische Physik, Universität zu Köln, Zülpicher Strasse 77, 50937 Cologne, Germany \\ (Received 25 November 2012; revised manuscript received 21 January 2013; published 29 March 2013)
}

\begin{abstract}
States supported by chaotic open quantum systems fall into two categories: a majority showing instantaneous ballistic decay, and a set of quantum resonances of classically vanishing support in phase space. We present a theory describing these structures within a unified semiclassical framework. Emphasis is put on the quantum diffraction mechanism which introduces an element of probability and is crucial for the formation of resonances. Our main result is boundary conditions on the semiclassical propagation along system trajectories. Depending on whether the trajectory propagation time is shorter or longer than the Ehrenfest time, these conditions describe deterministic escape, or probabilistic quantum decay.
\end{abstract}

DOI: 10.1103/PhysRevE.87.032918

PACS number(s): 05.45.Mt, 03.65.Sq, 03.65.Yz

\section{INTRODUCTION}

Quantum states populating "open" chaotic cavities decay to the outside environment and, thence, have the status of resonances. In spite of the ubiquity of the general setupopen quantum chaos is realized in many of the devices currently explored in mesoscopic physics, quantum optics, and cold atom physics-salient features of these resonances are not fully understood. While the deep quantum regime (the Ehrenfest time $t_{E}$, marking the diffractive disintegration of minimal wave packages shorter than classical escape times $t_{d}$ ) appears to be under reasonable control [1], it is the opposite, semiclassical limit which poses unsettled issues [2].

Broadly speaking, the states populating an open cavity can be grouped into two families: states evolving near classically and escaping deterministically after a classical flight time, and a fraction $\sim \exp \left(-t_{E} / t_{d}\right)$ of quantum resonances, whose probabilistic decay is characterized by a finite imaginary offset $i \Gamma / 2$ to the real resonance energy $E$. The most basic quantity characterizing the statistics of resonances of complex energy $z=E+i \frac{\Gamma}{2}$ is the resonance density $\rho(z)$. Although the quantitative profile of that quantity is not fully understood, the density appears to be gapped against the real axis, $\Gamma=0$ (the existence of rare midgap states notwithstanding [2,3].) The integrated number of resonances at a given value of $E$ has been found to obey the so-called fractal Weyl law, $\rho \propto \hbar^{-d_{f}}$, where $d_{f}$ is a nonuniversal fractal exponent.

Previous work on the phenomenon includes the formulation of lower bounds on the resonance gap [4-6], semiclassical approaches based on short periodic orbits trapped in the open system [7], a description in terms of nonunitarily evolving Husimi functions [8], phenomenology based on a mixture of phase-space dynamics and random matrix theory, respectively [9], and numerical analyses [2,5,8-12]. However, a unified theory of resonance formation in terms of first principle semiclassical dynamics appears to be missing and the formulation of such a theory is the subject of the present work.

Specifically, we will explore the quantum dynamics of states concentrated on classical trajectories in terms of phase-space Wigner functions. Assuming globally hyperbolic classical dynamics we will describe how quantum fluctuations operational on long trajectories convert the deterministic classical escape of short trajectories into probabilistic quantum decay.

Our analysis is organized in three conceptual steps. We first introduce the phase-space language used in the rest of the paper on a one-dimensional toy model (Sec. II). We then generalize to the more complex setting of a higher dimensional cavity (Sec. III), and derive effective boundary conditions determining the decay rates of the system. Finally, (Sec. IV) we analyze these equations for both short and long trajectories. We conclude in Sec. V.

\section{ONE-DIMENSIONAL TOY MODEL}

Consider a one-dimensional "cavity" parametrized by the spatial coordinate $q \in\left[-q_{0}, q_{0}\right]$, while coordinates to the right (left) of $+q_{0}\left(-q_{0}\right)$ define connecting "leads" (see Fig. 1). We assume free intracavity particle dynamics, $\hat{H}_{0}=\hat{p}^{2} / 2 m$ and, crucially, no backscattering barriers at the cavity-lead interfaces.

Life times and energies of the resonant states supported by the system may be calculated by matching solutions of the cavity Schrödinger equation to outgoing boundary conditions $[13,14]$, i.e., by requiring that cavity wave functions $\psi(q)$ and their derivatives smoothly connect to vacuum wave functions $\varphi_{ \pm}(q) \equiv a_{ \pm} \exp ( \pm i q \kappa)$ at the right or left interface. Here, $a_{ \pm}=$const, and $\kappa=k-i \frac{k_{\Gamma}}{2}$ is a complex wave vector whose real and imaginary part define the energy $\hbar k=(2 m E)^{1 / 2}$ and life time $\hbar k_{\Gamma} \equiv \Gamma / v$ of resonant states, respectively, where $v=\hbar k / m$. The divergence of the reference states at spatial infinity $q \rightarrow \pm \infty$, is a formal means [13] to the fixation of decay rates, as exemplified below.

For the intracavity wave function we make an ansatz $\psi(q)=\sum_{\sigma= \pm} a e^{\sigma\left[i \phi(q)+k_{\Gamma} v t(q) / 2\right]}$ in terms of left- and rightpropagating partial amplitudes where $a=$ const, and the somewhat unconventional denotation $\phi(q)=k q$ and $t(q)=$ $q / v$, for the real and imaginary contribution to the phase, respectively, will be motivated shortly. With this choice, the boundary conditions obtained by matching wave functions and their derivatives at the left and right interface reduce to the single algebraic equation

$$
e^{-\left[i 2 \phi\left(q_{0}\right)+k_{\Gamma} v t\left(q_{0}\right)\right]}=\left.\frac{\partial_{q} \phi-k-i \frac{k_{\Gamma}}{2}\left(v \partial_{q} t-1\right)}{\partial_{q} \phi+k-i \frac{k_{\Gamma}}{2}\left(v \partial_{q} t+1\right)}\right|_{q_{0}} .
$$


(a)

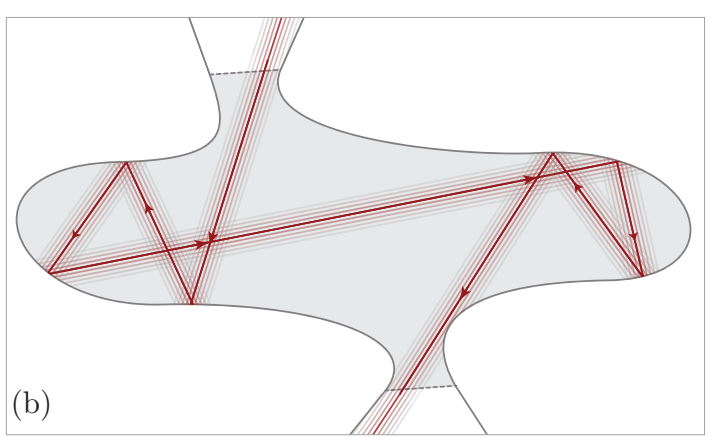

FIG. 1. (Color online) Schematic illustration of the models discussed in the text: (a) One-dimensional toy model consisting of a clean cavity (grey region) and leads connected to it (white regions). There is no backscattering at the cavity-lead interfaces and separation into cavity and leads is largely arbitrary. (b) Two-dimensional clean cavity with chaotic boundary scattering (grey region). Contact to the "outside world" is due to fully transmitting openings connecting to the reservoirs (white regions). Transitions from cavity to reservoirs are again smooth.

Before evaluating this equation, let us translate from the language of wave functions to a phase-space formulation. To this end, we introduce the Wigner function $W(q, p)=$ $\int(d a) e^{-i p a / \hbar} \bar{\psi}\left(q-\frac{a}{2}\right) \psi\left(q+\frac{a}{2}\right)$, where $(d a)=d a /(2 \pi \hbar)$. For our specific system,

$$
W(q, p)=\sum_{\sigma= \pm} a^{2} \delta[p-\sigma p(E)] e^{\sigma k_{\Gamma} v t(q)}+\cdots,
$$

where $\sigma= \pm$ labels the Wigner transform of the left and right moving components, respectively, and the ellipses denote rapidly oscillating interference contributions. In discarding the latter, we lose track of the global phase of the wave function, while the information on amplitudes and phase derivatives necessary to evaluate boundary conditions is retained. Indeed, it is straightforward to check that

$$
\begin{aligned}
a^{2} e^{\sigma k_{\Gamma} v t(q)} & =\left|\psi_{\sigma}(q)\right|^{2}=\int(d p) W_{\sigma}(q, p), \\
\sigma \hbar \partial_{q} \phi(q) & =\frac{\int(d p) p W_{\sigma}(q, p)}{\int(d p) W_{\sigma}(q, p)} .
\end{aligned}
$$

For the simple one-dimensional (1D) system, the linear dependence $\phi_{q}=k q$ implies $\partial_{q} \phi=k$ so that (1) reduces to

$$
\frac{8 E}{\Gamma} e^{-\Gamma t\left(q_{0}\right) / \hbar}=1-\left.v \partial_{q} t(q)\right|_{q=q_{0}},
$$

where we have "fixed a gauge" $e^{-2 i \phi\left(q_{0}\right)}=i$ for the arbitrary phase of the wave function and neglected contributions $k_{\Gamma} / k \ll 1$.

For the toy model at hand, $v \partial_{q} t(q)=1$, which means that the right hand side of (4) vanishes, and $\Gamma \rightarrow \infty$ is the only consistent solution. This reflects the fact that a wave function will "decay" with probability unity upon passing the reflectionless boundaries of the system. We next discuss how the situation changes upon generalization to a higher dimensional system with chaotic dynamics.

\section{CHAOTIC CAVITY}

We consider a two-dimensional cavity with ballistic Hamiltonian $\hat{H}=\hat{p}^{2} / 2 m$ and chaotic boundary scattering (see Fig. 2). The cavity is open such that after an average time $t_{d}$, much shorter than any of the relevant quantum time scales, trajectories escape through one or several reflectionless openings. We define the Wigner function of the system's resonance states by obvious generalization of Eq. (2), i.e., $W(\mathbf{q}, \mathbf{p})=\int\left(d^{2} a\right) e^{-i(\mathbf{p} \cdot \mathbf{a} / \hbar)} \bar{\psi}\left(\mathbf{q}-\frac{\mathbf{a}}{2}\right) \psi\left(\mathbf{q}+\frac{\mathbf{a}}{2}\right)$. To obtain the intracavity evolution equations of $W$, one adds and subtracts the Schrödinger equations of the resonances $\psi$ and $\bar{\psi}$ to obtain [15]

$$
\left[H^{*}, W\right]_{+}=2 E W, \quad\left[H^{*}, W\right]_{-}=-i \Gamma W .
$$

Here, $H=p^{2} / 2 m$ is the Hamilton function and $[A, B]_{\mp}=$ $A * B \mp B * A$ where the Moyal product of phase-space functions $A=A(\mathbf{q}, \mathbf{p})$ is given by [16] $A * B=A B+\frac{i \hbar}{2}\{A, B\}+$ $O\left(\hbar^{2}\right)$, and $\{$,$\} is the Poisson bracket.$

We next consider the vicinity of an exceptionally long trajectory $\gamma_{0}$ spending time $T \gg t_{d}$ inside the cavity. For completeness we note that long trajectories in open systems are found with low probability $\sim \exp \left(-T / t_{d}\right)$. They typically form in the phase-space neighborhood of strange repellers realized through periodic orbits trapped in the interior of the cavity (see below for further comments on this aspect). Assuming global hyperbolicity of the dynamics, we introduce a trajectory coordinate, $q \in[-v T / 2, v T / 2]$, a conjugate momentum $p=$ $p(H)=(2 m H)^{1 / 2}$ transverse to the shell of conserved energy, and a pair $u, s$ of locally unstable and stable coordinates. In the asymptotic neighborhood of $\gamma_{0}$, the Hamiltonian can then be approximated as $H \simeq H_{0}=\frac{p^{2}}{2 m}+\lambda u s$, where $\lambda$ is a Lyapunov exponent. The corresponding dynamics is generated by $\left[H_{0},{ }^{*}\right]_{-}=i \hbar\left\{H_{0},\right\} \equiv-i \hbar \mathcal{L}$, where the Liouvillian

$$
\mathcal{L}=v \partial_{q}+\lambda\left(u \partial_{u}-s \partial_{s}\right)
$$

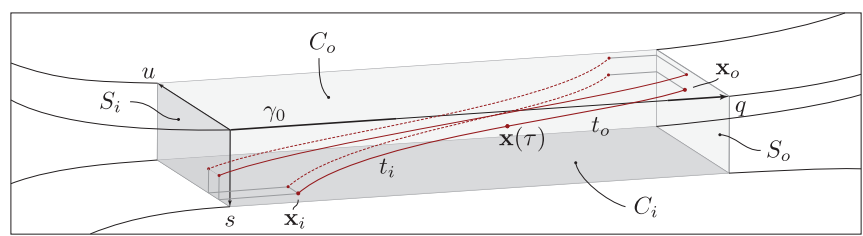

FIG. 2. (Color online) On the definition of the different time parameters relevant to the trajectory dynamics. A trajectory $\gamma_{0}$ (indicated by a straight line along the upper front corner of the box) enters or exits the system through phase-space interfaces $S_{i / o}$. Spanning the neighborhood of $\gamma_{0}$ by a stable (unstable) coordinate $u(s)$, nearby trajectories contract towards (depart from) $\gamma_{0}$ in the respective coordinate directions. The exit out of the cavity neighborhood of $\gamma_{0}$ then is through the system interface $S_{0}$ (solid trajectories), or through the "internal interface," $C_{o}$ (dashed trajectories.) A similar distinction applies to the entry points. Depending on the entry (exit) variants, each trajectory neighboring $\gamma_{0}$ gets assigned entry (exit) points $\mathbf{x}_{i}\left(\mathbf{x}_{o}\right)$, a timelike progression parameter $\tau$, and a phase-space parametrization $\mathbf{x}(\tau)$. 
describes propagation in the direction of $\gamma_{0}$, and exponential expansion (contraction) in the $u(s)$ coordinate. Nonlinear corrections to $H_{0}$ can be described as $H=H_{0}+V$, where $V=V(u, s)$ is a polynomial of degree $>2$ in the variables $u, s$. The corresponding modification of the dynamics, $\left[V^{*},\right]_{-} \equiv$ $-i \hbar(\Delta \mathcal{L}+\mathcal{Q})$, comprises a weak alteration of the classical Liouvillian, $\Delta \mathcal{L}$, and a quantum generator

$$
\mathcal{Q}=\sum_{n+m>1} c_{n m} \hbar^{n+m} \partial_{u}^{m} \partial_{s}^{n},
$$

where $c_{n m}=c_{n m}(q, u, s)$ are coefficient functions whose detailed profile will not be of much importance throughout. Although both contributions are nominally small in $u, s$, the quantum generator $\mathcal{Q}$, will be seen to have a regularizing effect on classical singularities [17], which will ultimately shape the profile of the resonance density.

\section{A. Life time in a chaotic cavity}

Close to the trajectory, the first of Eqs. (5), $\left[H^{*}, W\right]_{+} \simeq$ $\left[H_{0}, W\right]_{+} \simeq 2\left(p^{2} / 2 m\right) W=2 E W$, simply describes the onshell fixation $p \simeq(2 m E)^{1 / 2}$. Turning to the second equation,

$$
\hbar(\mathcal{L}+\Delta \mathcal{L}+\mathcal{Q}) W(q, u, s)=\Gamma W(q, u, s),
$$

we first discuss the linear approximation, $\Delta \mathcal{L}, \mathcal{Q}=0$, before including the correction terms in a second step.

For $\Delta \mathcal{L}, \mathcal{Q}=0,(8)$ becomes a first order differential equation which is solved in terms of a left- and a right-moving contribution,

$$
W(q, p, u, s)=\sum_{\sigma= \pm} a^{2} \delta[p-\sigma p(E)] e^{\sigma k_{\Gamma} v t(q, u, s)},
$$

structurally similar to Eq. (2). Here, $t(q, u, s)$ are effective parameter functions generalizing $t(q)$ of the toy model and evolving uniformly along the trajectories $\gamma \equiv \gamma_{\mathbf{x}}$ piercing the phase-space point $\mathbf{x} \equiv(q, u, s), \mathcal{L} t(\mathbf{x})=1$. To solve this (partial first order differential) equation, we consider its characteristics, i.e., the trajectory $\gamma_{\mathbf{x}}$. On $\gamma_{\mathbf{x}}$, the equation assumes the form $d_{\tau} t(\mathbf{x}(\tau))=1$, where $q(\tau)=q+v_{F} \tau, u(\tau)=u e^{\lambda \tau}$, $s(\tau)=s e^{-\lambda \tau}$, and $(q, u, s)$ are starting values of the evolution. We solve the characteristic equation as $t(\tau)=\tau+t^{0}$, where $\tau$ increases uniformly until $\gamma_{\mathbf{x}(\tau)}$ hits the effective boundaries of the problem, and $t^{0}$ is a freely adjustable parameter.

To understand the role of the boundaries, note that $\gamma_{\mathbf{x}}$ will leave the cavity either through a physical interface $S_{o}$ along with $\gamma_{0}=\gamma_{(q, 0,0)}$ (cf. Fig. 3), or it will depart from $\gamma_{0}$ up to some classical threshold $u \simeq c$ within the cavity (solid line). We assume that points separated from $\gamma_{0}$ by scales $\sim c$ have become generic and will exit in the classical, and hence negligibly short time $\simeq t_{d}$. The union $I_{o} \equiv S_{o} \cup C_{o}$ of $S_{o}$ and the surface $C_{o} \equiv\{u=c, s, q\}$ then defines the effective "outgoing interface" of our problem. Similarly, the union $I_{i} \equiv S_{i} \cup C_{i}$ of the left vacuum interface $S_{i}$ and the surface $C_{i}=\{u, s=c, q\}$ defines the incoming interface. The traveling phase-space point $\mathbf{x}(\tau)=(q, u, s)(\tau)$ hits the exit interface $I_{o}$, at the smaller of two times, $\tau=$ $t_{o}=t_{o}(q, u)=\min \left[\frac{T}{2}-\frac{q}{v}, \frac{1}{\lambda} \ln \left(\frac{c}{|u|}\right)\right]$, depending on whether $S_{o}$ or $C_{o}$ is the terminal. Likewise, $\gamma_{\mathbf{x}}$ has entered the cavity

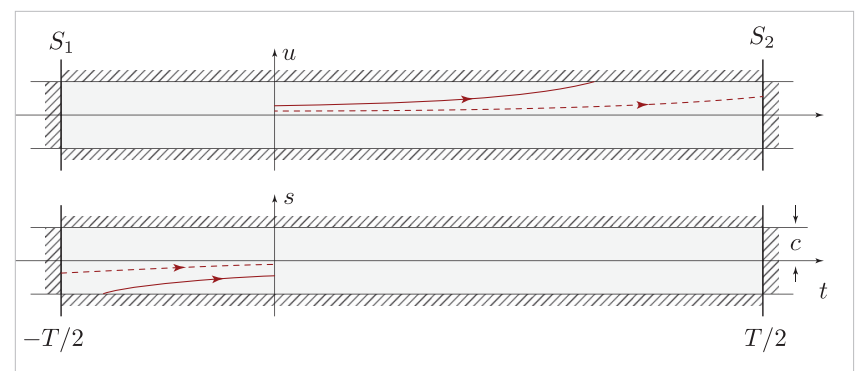

FIG. 3. (Color online) The information of Fig. 2 collapsed to the two-dimensional sections spanned by the stable and the unstable coordinate, and the trajectory parameter $q$, respectively. A phasespace point $(q, u, s)$ in the vicinity of $\gamma_{0}$ propagates along a unique classical trajectory $\gamma$. It will exit the cavity either through the interface $S_{o}$ or within the cavity through the surface $C_{o}$. Similarly, the union of the left vacuum interface $S_{i}$ together with the manifold $C_{i}$ defines the incoming interface.

through $I_{i}$ at a large negative time $\tau=-t_{i}=-t_{i}(q, s)=$ $-\min \left[\frac{T}{2}+\frac{q}{v}, \frac{1}{\lambda} \ln \left(\frac{c}{|s|}\right)\right]$. Fixing the free parameter $t^{0}$ such that the temporal range of the trajectory is symmetric around zero, $t\left(\tau=t_{i}\right)=-t\left(\tau=t_{o}\right)$, we find that the solution to Eq. (9) is governed by the function $t(\mathbf{x})=\frac{1}{2}\left[t_{i}(q, s)-t_{o}(q, u)\right]$ while $T_{\gamma} \equiv t_{i}+t_{o}$ is the intracavity flight time of $\gamma$. Notice that for points $\mathbf{x} \in I_{o}$ at the exit interface, $t_{o}(\mathbf{x})=0$, meaning that $t(\mathbf{x})=T_{\gamma} / 2$ attains its maximal value.

Finally, the boundary conditions Eq. (1) are generalized by replacing the one-dimensional variable $t(q)$ by $t(q, u, s)$, and the derivative $v \partial_{q}$ by $\mathcal{L}$, i.e., a derivative acting in the direction of the Hamiltonian flow [18]. The generalization of Eq. (1) thence reads

$$
\frac{8 E}{\Gamma} e^{-\Gamma t / \hbar}=1-\mathcal{L} t
$$

where $t=t(\mathbf{x})=T_{\gamma} / 2$, and $\mathbf{x} \in I_{o}$ is on the exit interface. Equation (10) is a principal result of the present paper. In the following we discuss its implications for different types of trajectories.

\section{DISCUSSION OF THE RESULTS}

\section{A. Short trajectories}

To start with, we consider trajectories $\gamma$ which are exceptionally long-lived, $T \equiv T_{\gamma} \gg t_{d}$, yet short in comparison to the scale $t_{E} \sim \frac{1}{\lambda} \ln \left(c^{2} / \hbar\right)$ [19] where quantum uncertainty leads to the disintegration of semiclassically propagating wave packets. As long as $T<t_{E}$, the quantum generator $\mathcal{Q}$ does not modify the dynamics in essential ways-a statement to be verified below-and the same goes for the generator of weak classical corrections, $\Delta \mathcal{L}$. We may thus take the boundary condition (10) at face value, and conclude that due to the homogeneous evolution $\mathcal{L} t=1$, the right hand side of the equation vanishes. As with the toy model, this implies a diverging decay rate, $\Gamma \rightarrow \infty$. In this divergence reflects the fact that wave packages traveling on classically short trajectories leave the cavity with certainty at the exit point. 


\section{B. Long trajectories}

We now turn to the case of long trajectories, $T>t_{E}$. For asymptotically long trajectories, $T \rightarrow \infty$, the classical shrinkage $s=s(t) \sim c \exp (-\lambda t)$ would lead to singularities in the function $t_{i}(q, s)$ and, ultimately, in the Wigner function. Within the present formalism, these singularities are regularized on time scales larger than $t_{E}$, where $s \lesssim s\left(t_{E}\right) \sim \hbar$ has shrunk down to quantum scales. On these scales, the quantum generator $\mathcal{Q}$ of Eq. (7) is no longer small in comparison to the classical generator $\mathcal{L}$ (while the correction $\Delta \mathcal{L}$ to the classical flow continues to be largely irrelevant). The ensuing modifications of the dynamics can be described in various ways (cf. Ref. [20] for a treatment tailored to the formalism applied here), the invariable conclusion being that the shrinkage of classically evolving variables gets cut off by quantum fluctuations. Technically, this conclusion rests on the observation that in the evolution equation for the variable $s$ the higher order derivatives $\sim \partial_{s}^{n>1}$ present in the quantum generator (7) build up "pressure" counteracting the classical contraction. This is seen in explicit terms in the Fourier or Laplace representation of the evolution equation, where these derivatives assume the form of algebraic factors, cutting the logarithmic "ultraviolet" singularities of the classical equation. Referring to the Appendix for more details, we note that to leading semiclassical accuracy functions which in the classical theory evolve as $f(q,|u|,|s|)$ get replaced by $f(q,|u|+\hbar / c,|s|+\hbar / c)$. Here, $c$ is symbolic notation for classical ( $\hbar$-independent) functions over which we have no explicit control, and the substitution $|u| \rightarrow|u|+\hbar$ becomes effectual in the large negative time asymptotics of a trajectory, where $u$ rather than $s$ scales to small values.

To understand the consequences of this regularization mechanism, consider the trajectory time parameter, $t=t_{i} / 2$ at the exit point of $\gamma$. Now notice that $t_{i}(q,|s|) \rightarrow \min [T / 2+$ $\left.q / v, \lambda^{-1} \ln (|s|+\hbar / c)\right]=\lambda^{-1} \ln (|s|+\hbar / c) \simeq t_{E}$, where we used that $T>t_{E}$. The crucial observation here is that the regularization effectively truncates the in-time function $t_{i}$ at values $t_{E}$. As a consequence, the interface derivative $\mathcal{L} t=$ $\frac{1}{2} \mathcal{L}\left(t_{i}-t_{o}\right)=1 / 2$ reduces to one-half of the value before quantum regularization. Substitution of this value into Eq. (10) shows that the quantum theory admits finite values of the decay constant, determined by

$$
\frac{\Gamma_{0}}{2}=\frac{\hbar}{t_{E}} W\left(\frac{8 E t_{E}}{\hbar}\right)=\frac{\hbar}{t_{E}}\left[\ln \left(\frac{8 E t_{E}}{\hbar}\right)+\cdots\right]
$$

where $W$ is the Lambert function and ellipses denote subleading double-"log" contributions. Equation (11) states the decay rate in terms of the Ehrenfest time in combination with nonuniversal short time cutoff $\hbar / E$. However, in the semiclassical limit, $\hbar \rightarrow 0$, the dependence on $E$ drops out, and we are left with the asymptote $\Gamma_{0} \sim \hbar \lambda$. Before commenting on this result, we note that the appearance of a finite decay rate within our present formalism follows from the fact that, by Heisenberg uncertainty, quantum mechanics is not capable of resolving the phase-space fine structures pertaining to the evolution of long trajectories $T_{\gamma}>t_{E}$. Each such trajectory should, rather, be thought of as a distribution defined by the union of trajectories with uncertainty $\sim \hbar$ in their phase-space coordinates. At a given instance of time, a fraction of this distribution escapes, as described by the rate $\Gamma_{0}$.

\section{Effective decay rate}

Our above analysis was oversimplifying in that it treated escape from an isolated long trajectory $\gamma_{0}$ as tantamount to escape into the lead vacuum. This picture ignores the fact that escaping trajectories may get "folded back" into the repeller domain supporting $\gamma_{0}$, and thence be trapped again. A statistical theory accounting for the renormalization of the decay of an initial distribution centered around an isolated trajectory by the complex structure of the embedding repeller structure has been developed in Refs. [4,21]. The result of that analysis is an effective renormalization of decay rates as $\lambda \rightarrow \lambda(1-d)$, where the factor $(1-d)$ effectively measures the fraction of trajectories managing to escape the repeller and $d$ is the fractal (information) dimension of the latter [4,22]. The ensuing effective rate, $\Gamma_{0} \rightarrow \Gamma \equiv \hbar \lambda(1-d)$, is generally identified with the inverse of the classical escape time of the system. We finally caution that the decay rate will be subject to sources of fluctuations which are beyond the scope of our analysis. Notably, the Lyapunov exponents may vary between trajectories, and along individual trajectories. The escape from the repeller may introduce additional uncertainty. Our result, thus, yields a characteristic value for the decay rate, where the important role of fluctuations is left unaccounted for. Other effects not captured by our analysis include transient features of the classical dynamics outside the repeller's area which, as recent work shows $[23,24]$, may have important influence on the resonances of open quantum system.

\section{Fractal Weyl law and random matrix regime}

For completeness we note that a finite quantum mechanical decay rate is attributed to states located in the vicinity of exceptionally long trajectory. Due to the exponential scarcity of these trajectories, the corresponding phase-space measure scales as [10] $n_{\Gamma}=\Omega_{E} e^{-t_{E} / t_{d}} \sim \Omega_{E} \hbar^{1 / t_{d} \lambda}$, where $\Omega_{E}$ is the phase-space volume of the energy shell (in units $\hbar$ ), implying that $n_{\Gamma} \sim \hbar^{-d_{f}}$ with fractal dimension $d_{f}=1-1 / \lambda t_{d}$ [25]. We finally note that in the quantum regime, $t_{E}<t_{d}$, which is complementary to the semiclassical regime $t_{d}<t_{E}$ studied here, random matrix scattering theory predicts [26] $\Gamma \sim$ $\frac{\hbar}{t_{d}} \ln \left(\tilde{E} t_{d} / \hbar\right)$, where $\tilde{E}$ is some cutoff energy scale. Comparison with (11) shows that the two results match at the boundary $t_{d} \sim t_{E}$. However, we cannot say whether or not this matching is coincidental.

\section{SUMMARY}

We have formulated a semiclassical theory of quantum escape processes in open chaotic systems. The most important single contribution of our approach is that it quantitatively describes how deterministic escape after the traversal of generic short trajectories through the system gives way to quantum mechanical decay on long trajectories. The latter define the support of resonances whose life times we estimated by imposing effective phase-space boundary conditions. Somewhat counterintuitively, it turns out that the ensuing decay rates are classically short $\Gamma_{0} \sim \hbar \lambda$, although the relevant escape 
dynamics takes place on long trajectories $T>t_{E}$. Finally, the escape of individual long trajectories as described in the present paper defines only an initializing stage of the decay of a more complicated repeller structure. As a result, the decay rate $\Gamma$ is subject to renormalization $\Gamma_{0} \rightarrow \Gamma=\hbar \lambda(1-d)$ where $d$ is the fractal repeller dimension. Qualitatively, the renormalization factor accounts for the probability that a state gets recaptured by the repeller structure after escaping an individual trajectory. However, a quantitative description of that secondary mechanism is beyond the scope of our approach.

\section{ACKNOWLEDGMENTS}

We thank P. W. Brouwer, S. Nonnenmacher, and $\mathrm{H}$. Schomerus for discussions and M. Novaes for pointing out an inconsistency in the interpretation of our results as given in the first version of this paper. Work was supported by SFB/TR 12 of the Deutsche Forschungsgemeinschaft.

\section{APPENDIX: REGULARIZATION}

We here discuss how quantum fluctuations regularize the unlimited classical contraction of the stable coordinate $s$ in a system with globally hyperbolic dynamics. In the language of Eqs. (6) and (7) of the main text, the dynamics of the variable $s$ is described by a differential equation of the structure

$$
\left(s \partial_{s}+\sum_{n \geqslant 1} c_{n} \hbar^{2 n+1} \partial_{s}^{2 n+1}\right) f(s)=-\alpha f(s),
$$

where $\alpha>0$, and in a manner inessential to the present argument the coefficients $c_{n}$ may depend on the variables $q, u, s$.

Considering positive starting values, $s>0$ (the extension to negative values is straightforward), we introduce a Laplace representation

$$
f(s)=\int_{0}^{\infty} d z e^{-s z} g(z)
$$

in which (A1) takes the form [27]

$$
\partial_{z} g(z)=-\left(\frac{1-\alpha}{z}+\sum_{n \geqslant 1} c_{n} \hbar^{2 n+1} z^{2 n}\right) g(z) .
$$

The general solution of this equation is found by straightforward integration over $z$, and when inserted into (A2) gives

$$
f(s)=c_{0} \int_{0}^{\infty} d z e^{-s z} z^{\alpha-1} e^{-\sum_{n \geqslant 1}\left[c_{n} /(2 n+1)\right](\hbar z)^{2 n+1}}
$$

with an integration constant $c_{0}$. Equation (A4) now illustrates the role played by higher differential operators in (A1).

To make the point, let us for the moment consider the first order differential equation obtained from (A1) by setting all $c_{n}=0$. The resulting function

$$
f^{0}(s)=c_{0} \int_{0}^{\infty} d z e^{-s z} z^{\alpha-1}=\frac{c_{0}}{s^{\alpha}}
$$

then displays the singular at small values of $s$ plaguing the classical evolution equation of the stable coordinate.

In the full solution Eq. (A4) the exponential factor $e^{-\sum_{n \geqslant 1}\left[c_{n} /(2 n+1)\right](\hbar z)^{2 n+1}}$ cuts the small-s or large- $z$ singularity at values $z \sim 1 / \hbar$. The resulting integral can be estimated by a regularized function

$$
f(s)=\frac{c_{0}}{(s+\hbar)^{\alpha}} .
$$

Finally, notice that our argument crucially relies on assumed positivity of the coefficients $c_{n}$. While the present construction cannot prove this feature, positivity is required on principal grounds to ensure stability of the dynamics. (Otherwise the Wigner distribution would cease to exist.) To actually demonstrate this stability, one has to work harder as in, e.g., Refs. [17,28]. A discussion tailored to the present formalism is contained in Ref. [20].
[1] Cf. Y. V. Fyodorov and H.-J. Sommers, Phys. A: Math. Gen. 36, 3303 (2003) for review.

[2] M. Novaes, J. Phys. A: Math. Theor. 46, 143001 (2013).

[3] M. Novaes, Phys. Rev. E 85, 036202 (2012).

[4] P. Gaspard and S. A. Rice, J. Chem. Phys. 90, 2225 (1989); 90, 2242 (1989).

[5] W. T. Lu, S. Sridhar, and M. Zworski, Phys. Rev. Lett. 91, 154101 (2003).

[6] S. Nonnenmacher and M. Zworski, Acta Math. 203, 149 (2009).

[7] M. Novaes, J. M. Pedrosa, D. Wisniacki, G. G. Carlo, and J. P. Keating, Phys. Rev. E 80, 035202(R) (2009).

[8] J. P. Keating, M. Novaes, S. D. Prado, and M. Sieber, Phys. Rev. Lett. 97, 150406 (2006).

[9] M. Kopp and H. Schomerus, Phys. Rev. E 81, 026208 (2010).

[10] H. Schomerus and J. Tworzydlo, Phys. Rev. Lett. 93, 154102 (2004).
[11] S. Nonnenmacher and M. Zworski, J. Phys. A: Math. Gen. 38, 10683 (2005).

[12] D. L. Shepelyansky, Phys. Rev. E 77, 015202(R) (2008),

[13] L. D. Landau and L. M. Lifshitz, Quantum Mechanics, NonRelativistic Theory (Pergamon Press, Oxford, 1977).

[14] N. Moiseyev, Non-Hermitean Quantum Mechanics (Cambridge University Press, Cambridge, England, 2011).

[15] W. P. Schleich, Quantum Optics in Phase Space (Cambridge University Press, Cambridge, 2011).

[16] J. E. Moyal, Proc. Cambridge Philos. Soc. Math. Phys. Sci. 45, 99 (1949).

[17] W. H. Zurek and J. P. Paz, Phys. Rev. Lett. 72, 2508 (1994).

[18] Notice that the appearance of $\mathcal{L}$ in this context does not imply a "classical approximation." Rather, the first order differential operator $\mathcal{L}$ describes the projection of the quantum current onto the outward direction of the dynamical flow at the cavity-lead interface. 
[19] A. I. Larkin and Yu. N. Ovchinikov, Zh. Eskp. Teor. Fiz. 55, 2262 (1968) [Sov. Phys. JETP 28, 1200 (1969)].

[20] T. Micklitz and A. Altland, Phys. Rev. Lett. 103, 080403 (2009).

[21] R. V. Jensen, Chaos 1, 101 (1991).

[22] E. Ott, Chaos in Dynamical Systems (Cambridge University Press, Cambridge, England, 2000).

[23] L. Ermann, G. G. Carlo, J. M. Pedrosa, and M. Saraceno, Phys. Rev. E 85, 066204 (2012).
[24] G. G. Carlo, D. A. Wisniacki, L. Ermann, R. M. Benito, and F. Borondo, Phys. Rev. E 87, 012909 (2013).

[25] Here we used $\Omega_{E}=L^{2} p / \hbar \ell$, where $L$ sets the size of cavity cavity and $\hbar / \ell$ the characteristic momentum resolution.

[26] H. Schomerus and P. Jacquod, J. Phys. A 38, 10663 (2005).

[27] For the sake of the argument, we focus on solutions subject to the condition $[z g(z)]_{z=0}=0$, such that (A3) does not contain a boundary term.

[28] A. Altland and F. Haake, Phys. Rev. Lett. 108, 073601 (2012). 